\title{
Epidemiología de las infecciones urinarias asociadas a catéter y no asociadas a catéter en un hospital universitario de tercer nivel
}

\author{
Diego F. Baenas, Emanuel J. Saad, Fernando A. Diehl, Danilo Musso, \\ Jacqueline G. González, Victoria Russo, Mario Vilaró y Ricardo A. Albertini
}

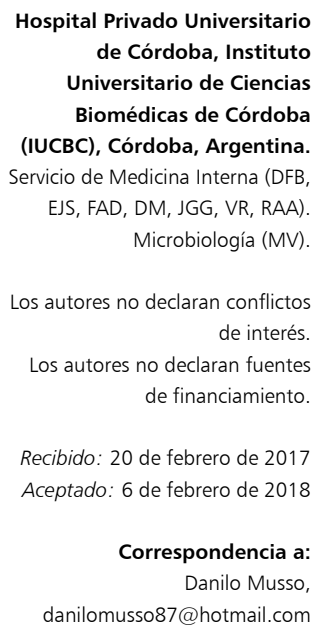

Recibido: 20 de febrero de 2017 Aceptado: 6 de febrero de 2018

Correspondencia a: Danilo Musso, danilomusso87@hotmail.com

\section{Nosocomial urinary tract infection: an analysis beyond urinary catheterization}

Introduction: Nosocomially acquired urinary tract infections (NAUTI) represent an important public health issue, but its characteristics when they are not catheter associated (CA-UTI) or when they take place outside intensive care units (ICU) are poorly understood. Objectives: To determine the patients' characteristics, etiology and antimicrobial susceptibility of NAUTI, both CA-UTI and no CA-UTI, in general ward and ICU. Methods: We conducted a retrospective analytic cross-sectional study, between 2009 and 2013, in a third level universitary hospital. All NAUTI episodes were identified, classifying them as CA-UTI and no CA-UTI. Results: We included 253 episodes of NAUTI, being CA-UTI (60,9\%) more frequent than no CA-UTI. A 37,4\% of no CA-UTI and 59,7\% of CA-UTI were identified in ICU. The most frequently isolated microorganisms were Escherichia coli, Klebsiella pneumoniae and Enterococcus sp. A $19 \%$ of extended spectrum betalactamase producing gram negative bacilli were found, without differences between groups. Conclusion: Patients's comorbidities, microorganisms associated to NAUTI and its antimicrobial susceptibility were similar in CA-UTI and no CA-UTI, as in general ward and ICU.

Key words: Urinary tract infections, cross infection, urinary catheters, intensive care units.

Palabras clave: Infecciones urinarias, infección hospitalaria, catéteres urinarios, unidad de cuidados intensivos.

\section{Introducción}

L as infecciones urinarias asociadas a la atención de la salud (ITU-AAS) representan un importante problema de salud pública, estando asociadas a incremento de la morbimortalidad de los pacientes, días de internación y costos en salud ${ }^{1-7}$. Múltiples reportes mencionan a las infecciones urinarias como la infección más frecuente dentro del ámbito hospitalario ${ }^{6,8,9}$.

El conocimiento de los factores de riesgo asociados y el perfil de susceptibilidad de los microorganismos causantes de las mismas son herramientas indispensables para generar guías de tratamiento adecuadas. Clásicamente se ha postulado a la cateterización urinaria permanente como el principal factor asociado al desarrollo de las ITU-AAS, debido a que la formación de biopelículas dentro de la superficie del catéter favorece el crecimiento de los microorganismos y su resistencia a los antimicrobianos ${ }^{5}$. Por esta razón, la mayoría de los estudios han centrado su análisis en pacientes portadores de catéter urinario permanente e internados en unidades de cuidados intensivos (UCI) ${ }^{1-5}$. Sin embargo, diferentes autores han reportado que entre 20 y $40 \%$ de las ITUAAS no estaban asociadas a catéter ${ }^{6,10,11}$. Por ello, el análisis de otros posibles factores de riesgo además de la cateterización urinaria, adquiere gran importancia a la hora de abordar el desarrollo de ITU-AAS. Entre ellos, los más frecuentemente identificados han sido la duración de la estancia hospitalaria (particularmente en UCI), diabetes mellitus, edad (mayores de 60 años), antecedente de patología estructural de la vía urinaria, inmunosupresión y el tratamiento antimicrobiano previo $^{3,5,8,10,12}$. A pesar de ello, los datos sobre las ITU-AAS fuera de UCI, así como de aquellas que no se relacionan a la cateterización urinaria siguen siendo limitados. En la última década, en nuestro país sólo se han reportado publicaciones aisladas en relación a la incidencia de ITU-AAS ${ }^{13-16}$.

El objetivo del presente trabajo fue establecer las características epidemiológicas de las infecciones urinarias nosocomiales asociadas y no asociadas a catéter urinario permanente.

\section{Materiales y Métodos}

Se llevó a cabo un estudio analítico, retrospectivo, de corte transversal, en un hospital universitario de tercer nivel de atención, ubicado en la ciudad de Córdoba, Argentina (Hospital Privado Universitario de Córdoba). 
Esta institución posee 206 camas y un promedio de 12.890 admisiones anuales. El Laboratorio de Microbiología de la institución cuenta con una base de datos donde se registran todos los estudios de urocultivos (UC) realizados. A partir de la misma, se identificaron todos los UC informados como positivos en pacientes mayores de 18 años de edad, durante el período comprendido entre agosto de 2009 y enero de 2013. Mediante la revisión de las historias clínicas electrónicas de los pacientes con UC positivos, se identificaron aquellos que cumplían con criterios clínicos y analíticos para ITU-AAS según Centers for Disease Control and Prevention (CDC) de Atlanta, E.U.A. Posteriormente, se los clasificó en infección de tracto urinario nosocomial asociada a catéter (ITU-C) e infección de tracto urinario nosocomial no asociada a catéter (ITU-noC). De cada uno de estos casos, se registró en una tabla diseñada específicamente para este estudio, las características demográficas, días de estancia hospitalaria en los 30 días previos al episodio, área de internación (UCI o sala general), uso de antimicrobianos durante los tres meses previos al episodio, antecedente de infecciones urinarias a repetición, co-morbilidades asociadas (trasplante renal, alteración de la vía urinaria, diabetes mellitus) y uso de inmunosupresores. En el caso de las ITU-C, se corroboró que todas las muestras de UC hubiesen sido tomadas previo recambio de catéter vesical, de acuerdo con la normativa vigente en el hospital.

\section{Definiciones}

ITU-AAS, ITU-C, ITU- noC e ITU a repetición: fueron determinadas de acuerdo a las definiciones clásicas ${ }^{11,17-19}$.

Alteración de la vía urinaria: Alteraciones orgánicas, funcionales o estructurales de la vía urinaria, o la presencia de dispositivos en la misma ${ }^{20}$. Instrumentación de la vía urinaria en los últimos 90 días: Colocación de catéteres permanentes, procedimientos endo-urológicos (ureteroscopia, ureterocistoscopia, urografía retrógrada, nefrostomía percutánea o cualquier procedimiento quirúrgico que alterase la anatomía del árbol urinario) realizados entre los últimos 90 días y $48 \mathrm{~h}$ previo a la toma del urocultivo que definió el episodio de ITU-AAS.

El Laboratorio de Microbiología utilizó como método de rutina la técnica automatizada de Vitek ${ }^{\circledR}$ para determinar la identificación de especie y también la susceptibilidad antimicrobiana in vitro, a través de la concentración inhibitoria mínima. El laboratorio se encuentra integrado al programa de control de calidad externo de pruebas de sensibilidad a antimicrobianos del Instituto de Salud "Dr. Carlos Malbrán”. Se relevaron los espectros de resistencia a los antimicrobianos más frecuentemente utilizados en el hospital.

Estudio aprobado por el Comité Institucional de Ética de Investigación en Salud de nuestra institución.

\section{Análisis estadístico}

Las variables continuas se expresaron como media y desviación estándar y la comparación de las mismas se realizó con test t de Student o Mann-Whitney de acuerdo a su homogeneidad. Las variables categóricas se expresaron como número y porcentaje, y se analizaron con test de $\chi^{2}$ o exacto de Fisher de acuerdo a las frecuencias esperadas. Se consideró como significativo un valor de probabilidad $<0,05$. El análisis estadístico se realizó con el programa estadístico SPSS 17,0 (SPSS, Inc., Chicago, IL).

\section{Resultados}

Durante el período de estudio se reportó un total de 2.822 UC positivos, de los cuales 253 se dieron en un contexto clínico que cumplía con los criterios de inclusión de ITU-AAS. De éstos, 60,9\% correspondió a ITU-C y $39,1 \%$ a ITU-noC.

La edad promedio fue de 63 años. Del total de las ITU-AAS, 50,2\% ocurrió en pacientes de sexo masculino, presentando mayor frecuencia dentro de las ITU-C $(\mathrm{p}<0,001)$.

\section{Factores de riesgo}

Se observó que 79,9 y 66,7\% de los pacientes con ITUC e ITU-noC, respectivamente, habían recibido antimicrobianos dentro de los treinta días previos al diagnóstico $(p=0,018)$. La media de días que estuvieron internados los pacientes durante el mes previo al desarrollo de ITUAAS, fue mayor en ITU-C $(p=0,013)$. En un sub-análisis de días de internación en ambos grupos, se observó que $38,4 \%$ de las ITU-noC y $21,1 \%$ de las ITU-C estuvieron internados entre 2 y 5 días $(\mathrm{p}<0,001)$ (Tabla 1$)$.

El principal agente etiológico aislado de las ITU-AAS fue Escherichia coli $(43,9 \%)$, siendo más frecuente en el grupo de las ITU-noC (53,5 vs 37,7\%, p =0,013), seguido de Klebsiella pneumoniae (20,6\% del total, representando $24,7 \%$ de las ITU-C y $14,1 \%$ de las ITU-noC), Enterococcus sp (7,9\% del total de aislados), Pseudomonas aeruginosa (7,5\% del total de aislados) y Acinetobacter baumannii (5,9\% del total de aislados).

En cuanto a los perfiles de sensibilidad antimicrobiana de los agentes etiológicos de ambos grupos, sólo se observó diferencia significativa en imipenem, con mayor resistencia en el grupo ITU-C (12,1\% vs $3,5 \%, p=0,03)$. Sobre el total de las ITU-AAS, se aislaron 48 UC positivos por microorganismos productores de $\beta$-lactamasa de espectro extendido (BLEE) (19\%), representando porcentajes similares entre los episodios de ITU-noC e ITU-C (Tabla 2).

En la Tabla 3 se comparan los perfiles de susceptibilidad de los bacilos gramnegativos más frecuentemente aislados (ITU-C vs ITU-noC). Más de $20 \%$ de las cepas de $E$. coli fueron resistentes a ampicilina/sulbactam, cefazolina, 
Tabla 1. Características de los pacientes con infección urinaria asociada a la atención de salud, asociada y no asociada a catéter urinario permanente

\begin{tabular}{|c|c|c|c|}
\hline & $\begin{array}{l}\text { ITU-noC } \\
(n=99)\end{array}$ & $\begin{array}{c}\text { ITU-C } \\
(n=154)\end{array}$ & $\mathbf{p}$ \\
\hline $\begin{array}{l}\text { Sexo masculino, } n(\%) \\
\text { Edad, } \bar{x} \pm \text { DE (años) }\end{array}$ & $\begin{array}{c}35(35,4) \\
61,4 \pm 19,6\end{array}$ & $\begin{array}{c}92(59,7) \\
63,6 \pm 18,3\end{array}$ & $\begin{array}{r}<0,001 \\
0,361\end{array}$ \\
\hline $\begin{array}{l}\text { Número de días internados en los últimos } 30 \text { días } \\
\text { Días de internación, } \mathrm{X} \pm \mathrm{DE} \\
\text { Internados entre 2-5 días, } \mathrm{n}(\%) \\
\text { Internados entre 6-10 días, } \mathrm{n}(\%) \\
\text { Internados entre } 11-20 \text { días, n (\%) } \\
\text { Internados entre 21-30 días, n (\%) }\end{array}$ & $\begin{array}{l}10,47 \pm 8,5 \\
38(38,4) \\
26(26,4) \\
19(19,2) \\
16(16,2)\end{array}$ & $\begin{array}{ll}13,3 & \pm 8,9 \\
31 & (20,1) \\
49 & (31,8) \\
40 & (26) \\
34 & (22,1)\end{array}$ & $\begin{array}{l}0,013 \\
0,001 \\
0,345 \\
0,213 \\
0,249\end{array}$ \\
\hline $\begin{array}{l}\text { Co-morbilidades } \\
\text { Litiasis urinaria, n (\%) } \\
\text { Diabetes mellitus, n (\%) } \\
\text { Alteración urológica, n (\%) } \\
\text { ITU recurrente, n (\%) }\end{array}$ & $\begin{array}{rr}5 & (5,1) \\
24 & (24,2) \\
39 & (39,4) \\
14 & (14,3)\end{array}$ & $\begin{array}{rr}3 & (1,9) \\
41 & (26,6) \\
64 & (41,6) \\
13 & (8,4)\end{array}$ & $\begin{array}{l}0,269 \\
0,672 \\
0,732 \\
0,152\end{array}$ \\
\hline $\begin{array}{l}\text { Otros factores de riesgo } \\
\text { Antimicrobianos en el último mes, n (\%) } \\
\text { Internación en } \mathrm{UCl}, \mathrm{n}(\%) \\
\text { Quimioterapia, } \mathrm{n}(\%) \\
\text { Trasplante, } \mathrm{n}(\%) \\
\text { Trasplante renal, } \mathrm{n}(\%) \\
\text { Instrumentación de la vía urinaria en los últimos } 90 \text { días, n (\%) } \\
\text { Institucionalizado, } \mathrm{n}(\%)\end{array}$ & $\begin{array}{l}66(66,7) \\
37(37,4) \\
11(11,1) \\
31(31,3) \\
27(27,3) \\
53(53,5) \\
3(3)\end{array}$ & $\begin{array}{rr}123 & (79,9) \\
92 & (59,7) \\
10 & (6,5) \\
35 & (22,7) \\
32 & (20,8) \\
154 & (100) \\
& 6(3,9)\end{array}$ & $\begin{array}{r}0,018 \\
0,001 \\
0,194 \\
0,129 \\
0,233 \\
<0,001 \\
1\end{array}$ \\
\hline
\end{tabular}

ITU: Infección del tracto urinario; ITU-C: Infección urinaria asociada a catéter urinario permanente. ITU-noC: Infección urinaria no asociada a catéter urinario permanente; UCI: Unidad de cuidados intensivos.

Tabla 2. Principales aislamientos microbiológicos de las infecciones urinarias asociadas a la atención de la salud y sus perfiles de resistencia

\begin{tabular}{|c|c|c|c|}
\hline Microorganismos aislados & $\begin{array}{c}\text { ITU-noC }(n=99) \\
n(\%)\end{array}$ & $\begin{array}{c}\text { ITU-C }(n=154) \\
n(\%)\end{array}$ & $\mathbf{p}$ \\
\hline $\begin{array}{l}\text { Bacilos gramnegativos } \\
\text { Escherichia coli } \\
\text { Klebsiella pneumoniae } \\
\text { Pseudomonas aeruginosa } \\
\text { Acinetobacter baumannii } \\
\text { Proteus mirabilis } \\
\text { Enterobacter cloacae } \\
\text { Serratia marcescens } \\
\text { Citrobacter }\end{array}$ & $\begin{array}{cc}53 & (53,5) \\
14 & (14,1) \\
8 & (8,1) \\
2 & (2) \\
4 & (4) \\
1 & (1) \\
3 & (3) \\
1 & (1)\end{array}$ & $\begin{aligned} 58 & (37,7) \\
38 & (24,7) \\
11 & (7,2) \\
13 & (8,5) \\
5 & (3,2) \\
7 & (4,5) \\
5 & (3,3) \\
3 & (2)\end{aligned}$ & $\begin{array}{l}0,013 \\
0,043 \\
0,810 \\
0,035 \\
0,740 \\
0,154 \\
1 \\
1\end{array}$ \\
\hline $\begin{array}{l}\text { Cocos grampositivos } \\
\text { Enterococcus sp } \\
\text { Staphylococcus coagulasa negativa } \\
\text { Staphylococcus aureus }\end{array}$ & $\begin{array}{cc}11 & (11,1) \\
2 & (2) \\
0 & (0)\end{array}$ & $\begin{array}{ll}9 & (5,8) \\
3 & (1,9) \\
2 & (1,3)\end{array}$ & $\begin{array}{l}0,154 \\
1 \\
0,522\end{array}$ \\
\hline $\begin{array}{l}\text { Resistencia antimicrobiana } \\
\text { Ampicilina/sulbactam } \\
\text { Cefalotina } \\
\text { Cefepime } \\
\text { Cefotaxima } \\
\text { Ciprofloxacina } \\
\text { Gentamicina } \\
\text { Imipenem } \\
\text { Piperacilina/tazobactam } \\
\text { Vancomicina } \\
\text { Colistina } \\
\text { Amikacina } \\
\text { Ceftazidima } \\
\text { Microorganismos productores de BLEE }\end{array}$ & $\begin{array}{l}54(64,3) \\
43(57,3) \\
25(29,1) \\
21(29,2) \\
47(47,5) \\
30(33,3) \\
3 \quad(3,5) \\
20(23,3) \\
3(21,4) \\
0(0) \\
3(3,59) \\
1(11,1) \\
17(17,2)\end{array}$ & $\begin{array}{l}87(65,9) \\
64(56,1) \\
48(61,4) \\
41(38) \\
62(40,5) \\
51(34,9) \\
17(12,1) \\
42(30,2) \\
0(0) \\
0(0) \\
10(7,1) \\
7(38,9) \\
31(20,1)\end{array}$ & $\begin{array}{l}0,807 \\
0,871 \\
0,355 \\
0,224 \\
0,277 \\
0,802 \\
0,030 \\
0,256 \\
0,09 \\
1 \\
0,379 \\
0,201 \\
0,558\end{array}$ \\
\hline
\end{tabular}




\begin{tabular}{|c|c|c|c|c|c|c|c|c|c|}
\hline & \multicolumn{2}{|c|}{ E. coli $(n=111)$} & \multirow[b]{2}{*}{$\mathbf{p}$} & \multicolumn{2}{|c|}{ K. pneumoniae $(n=52)$} & \multicolumn{3}{|c|}{$P$. aeruginosa $\quad(n=19)$} & \multirow[b]{2}{*}{$\mathbf{p}$} \\
\hline & $\begin{array}{c}\text { ITU-C ( } n=58) \\
n(\%)\end{array}$ & $\begin{array}{c}\text { ITU-noC }(n=53) \\
n(\%)\end{array}$ & & $\begin{array}{c}\text { ITU-C ( } n=38) \\
n(\%)\end{array}$ & $\begin{array}{c}\text { ITU-noC }(n=14) \\
n(\%)\end{array}$ & $\mathbf{p}$ & $\begin{array}{c}\text { ITU-C }(n=11) \\
n(\%)\end{array}$ & $\begin{array}{c}\text { ITU-noC }(n=8) \\
n(\%)\end{array}$ & \\
\hline AMS & $36(62,1)$ & $34(64,1)$ & 0,82 & $27(71)$, & $12(85,7)$ & 0,28 & NA & NA & NA \\
\hline Cefazolina & $31(53,4)$ & $29(54,7)$ & 0,89 & $22(57,9)$ & $8(57,1)$ & 0,96 & NA & NA & NA \\
\hline Ciprofloxacina & $24(41,4)$ & $24(45,3)$ & 0,68 & $12(31,6)$ & $7(50)$ & 0,22 & $4(36,4)$ & $3(37,5)$ & 1 \\
\hline Cefepime & $11(19)$ & $9(17)$ & 0,79 & $16(42,1)$ & $8(57,1)$ & 0,33 & $1(9,1)$ & $1(12,5)$ & 1 \\
\hline Ceftriaxona & $16(27,6)$ & $11(20,7)$ & 0,4 & $17(44,7)$ & $8(57,1)$ & 0,43 & NA & NA & NA \\
\hline PTZ & $6(10,3)$ & $6(11,3)$ & 0,87 & $17(44,7)$ & $9(64,3)$ & 0,21 & $1(9,1)$ & NA & 1 \\
\hline Imipenem & $0 \quad(0)$ & $0 \quad(0)$ & $0(0)$ & $2(5,3)$ & $1 \quad(7,1)$ & 0,8 & $2(18,2)$ & $0 \quad(0)$ & 0,48 \\
\hline Gentamicina & $14(24,1)$ & $13(24,5)$ & 0,96 & $13(34,2)$ & $8(57,1)$ & 0,13 & $2(18,2)$ & $1(12,5)$ & 1 \\
\hline Amikacina & $1 \quad(1,7)$ & $1 \quad(1,9)$ & 0,95 & $2 \quad(5,3)$ & $1 \quad(7,1)$ & 0,8 & $2(18,2)$ & $0 \quad(0)$ & 0,48 \\
\hline
\end{tabular}

ceftriaxona, ciprofloxacina y gentamicina. La mayoría de los aislados de K. pneumoniae, fueron resistentes a todos los antimicrobianos testeados, excepto a imipenem y amikacina. Estos hallazgos se observaron en ambos grupos, sin poder establecer una diferencia significativa entre ambos.

Por último, en lo que respecta al grupo de Enterococcus, $27,3 \%$ de dichos aislados de ITU-noC fueron resistentes a vancomicina.

Acinetobacter baumannii fue aislado en 15 episodios de ITU-AAS (86,7\% de estos en el grupo de ITU-C), tratándose en su totalidad de cepas multi-resistentes, con una sensibilidad de $100 \%$ a colistín.

El número de eventos de ITU nosocomiales que ocurrieron en sala general y en UCI fue similar. Al comparar las características entre ambos grupos, se observó que el antecedente de catéter permanente fue más frecuente en pacientes internados en UCI. Los episodios en sala general se caracterizaron por presentarse más frecuentemente en pacientes con alteración urológica, quimioterapia o ser receptor de trasplante renal (Tabla 4).

\section{Discusión}

Nuestro estudio aborda la problemática de las infecciones urinarias nosocomiales desde una visión diferente a la publicada previamente. Se ha considerado a todas las ITU-AAS, sin excluir las ITU-noC ni a los eventos ocurridos fuera de UCI. En nuestra institución, 39,1\% de las ITU-AAS fueron ITU-noC, constituyendo un porcentaje no despreciable dentro de las ITU-AAS. Dentro de la poca bibliografía que ha comparado ambos tipos de ITU-AAS, se ha reportado una frecuencia cercana a $30 \%$ de ITUnoC ${ }^{7,10}$. De este modo, se denota la importante frecuencia de dichas infecciones en nuestra institución, adquiriendo un valor epidemiológico de gran importancia.

Los factores de riesgo más comúnmente identificados de ITU-AAS, al igual que otros estudios, han sido el uso de antimicrobianos en el último mes, cateterización urinaria, ITU en el año previo y diabetes mellitus ${ }^{10,21-23}$. Cabe destacar que otras co-morbilidades frecuentes de los pacientes de nuestro estudio fueron el antecedente de haber recibido trasplante renal y la pre-existencia de alteración urológica. Al comparar factores de riesgo entre los grupos ITU-C e ITU-noC, se observaron diferencias significativas en la utilización de antimicrobianos en el último mes, antecedente de instrumentación de la vía urinaria y en el número de días de internación en el último mes, que fueron mayores en los pacientes con ITU-C.

A diferencia de otros trabajos, en los cuales la frecuencia de ITU-AAS ha sido mayor en UCI que en sala general $^{10,24}$ en nuestro estudio no se observaron diferencias significativas entre las infecciones ocurridas en ambos servicios. Esto demuestra la importancia de considerar las ITU-AAS en todas las áreas del hospital y no sólo en UCI, teniendo grandes implicancias en la morbimortalidad de los pacientes y costos sanitarios.

Coincidiendo con lo comunicado en múltiples publicaciones nacionales e internacionales, E. coli fue el agente más frecuentemente aislado, causando $43,9 \%$ de ITU-AAS, seguido por K. pneumoniae, Enterococcus sp y $P$. aeruginosa ${ }^{1-5,8,25-27}$.

Al considerar los perfiles de susceptibilidad a antimicrobianos de los microorganismos aislados, éstos fueron similares en los grupos de ITU-C e ITU-noC, observándose sólo una mayor resistencia a imipenem en el primer grupo. Esto último podría deberse a que $A$. baumannii fue el principal microorganismo resistente a imipenem y su aislamiento estuvo relacionado a las ITU-C. 


\begin{tabular}{|c|c|c|c|}
\hline $\begin{array}{l}\text { Características de los pacientes y } \\
\text { principales aislamientos }\end{array}$ & $\begin{array}{l}\text { Sala General }(n=124) \\
n(\%)\end{array}$ & $\begin{array}{l}\mathrm{UCI}(\mathrm{n}=129) \\
\mathrm{n}(\%)\end{array}$ & p \\
\hline Sexo masculino & $61(49,2)$ & $66(51,2)$ & 0,754 \\
\hline Antimicrobiano en el último mes & $88(71)$ & $101(78,3)$ & 0,180 \\
\hline Antimicrobiano en los últimos 90 días & $86(69,4)$ & $92(71,3)$ & 0,733 \\
\hline ITU-C & $62(50)$ & $92(71,3)$ & 0,001 \\
\hline Diabetes mellitus & $33(26,6)$ & $32(24,8)$ & 0,742 \\
\hline Litiasis urinaria & $4(3,2)$ & $4(3,1)$ & 1 \\
\hline Alteración urológica & $68(54,8)$ & $35(27,1)$ & $<0,001$ \\
\hline Quimioterapia & $15(12,1)$ & $6(4,7)$ & 0,032 \\
\hline Trasplante de órgano sólido & $42(33,9)$ & $24(18,6)$ & 0,006 \\
\hline Trasplante renal & $41(33,1)$ & $18(14)$ & $<0,001$ \\
\hline ITU recurrente & $19(15,3)$ & $8(6,2)$ & 0,019 \\
\hline Catéter en los últimos 90 días & $89(71,8)$ & $118(91,5)$ & $<0,001$ \\
\hline Institucionalizado & $6(4,8)$ & $3(2,3)$ & 0,327 \\
\hline \multicolumn{4}{|l|}{ Microorganismos } \\
\hline Citrobacter sp & $3(2,4)$ & $1(0,8)$ & 0,362 \\
\hline Enterococcus sp & $15(12,1)$ & $5(3,9)$ & 0,015 \\
\hline Escherichia coli & $64(51,6)$ & $47(36,4)$ & 0,015 \\
\hline Klebsiella pneumoniae & $16(12,9)$ & $36(27,9)$ & 0,003 \\
\hline Proteus mirabilis & $4(3,2)$ & $5(3,9)$ & 1 \\
\hline Pseudomonas aeruginosa & $9(7,3)$ & $10(7,8)$ & 0,882 \\
\hline Serratia marcescens & $1(0,8)$ & $7(5,4)$ & 0,066 \\
\hline Staphylococcus aureus & $2(1,6)$ & $0(0)$ & 0,239 \\
\hline Acinetobacter baumannii & $2(1,6)$ & $13(10,1)$ & 0,006 \\
\hline Enterobacter cloacae & $4(3,2)$ & $4(3,1)$ & 1 \\
\hline Staphylococcus coagulasa negativa & $3(2,4)$ & $2(1,6)$ & 0,679 \\
\hline \multicolumn{4}{|l|}{ Resistencia antimicrobiana } \\
\hline Ampicilina/sulbactam & $63(59,4)$ & $78(70,9)$ & 0,077 \\
\hline Cefalotina & $52(54,2)$ & $55(59,1)$ & 0,490 \\
\hline Cefepime & $22(21,8)$ & $51(41,8)$ & 0,002 \\
\hline Cefotaxima & $25(28,1)$ & $37(40,7)$ & 0,079 \\
\hline Ciprofloxacina & $52(42,3)$ & $57(44,2)$ & 0,760 \\
\hline Gentamicina & $34(30,6)$ & $47(37,6)$ & 0,260 \\
\hline Imipenem & $3(2,9)$ & $17(13,9)$ & 0,004 \\
\hline Piperacilina/tazobactam & $23(22,3)$ & $39(32)$ & 0,107 \\
\hline Vancomicina & $3(13,6)$ & $0(0)$ & 0,545 \\
\hline Colistina & $0(0)$ & $0(0)$ & 1 \\
\hline AmiKacina & $3(2,9)$ & $10(8,2)$ & 0,150 \\
\hline Ceftazidima & $4(30,8)$ & $4(28,6)$ & 1 \\
\hline Microorganismos productores de BLEE & $15(12,1)$ & $33(25,6)$ & 0,006 \\
\hline
\end{tabular}

UCI: Unidad de cuidados intensivos; ITU: Infección del tracto urinario; ITU-C: Infección del tracto urinario asociada a catéter urinario permanente; ITU recurrente: Infección del tracto urinario recurrente. BLEE: $\beta$-lactamasa de espectro extendido. 
Teniendo en cuenta los perfiles de susceptibilidad de los tres microorganismos gramnegativos más frecuentes (que representaron $72 \%$ de la muestra) entre los grupos de ITU-C e ITU-noC, no se observaron diferencias significativas. Este hallazgo reviste gran importancia, ya que la presencia de catéter urinario no debería modificar la conducta terapéutica a la hora de instaurar un tratamiento empírico para infecciones urinarias nosocomiales. Si bien ciprofloxacina es uno de los principales antimicrobianos empleado para el tratamiento empírico de las ITU adquiridas en la comunidad, en el ámbito nosocomial hemos observado una resistencia a dicho antimicrobiano de $43 \%$, sin diferencias entre ITU-C e ITU-noC. Estos hallazgos son similares a los reportados en un gran meta-análisis que incluyó estudios de múltiples países, reportando una tasa de resistencia de $38 \%$, la que era mayor en regiones en desarrollo ${ }^{28}$.

En nuestro relevamiento, $19 \%$ del total de microorganismos aislados fueron productores de BLEE, lo que contrasta con otros estudios en que la proporción de microorganismos productores de BLEE fue mayor ${ }^{29}$. El $17,1 \%$ de las cepas de $E$. coli y $36,5 \%$ de las cepas de $K$. pneumoniae fueron productoras de BLEE, valores similares a los observados en estudios realizados en Europa, pero mayores a los reportados en América del Norte ${ }^{30}$. Un dato a destacar es que no observamos diferencias en la frecuencia de eventos causados por microorganismos productores de BLEE entre ITU-C e ITU-noC. Sin embargo, se observó que la mayoría los pacientes en que se aislaron microorganismos productores de BLEE tenían antecedente de internación en UCI. Al realizar un sub-análisis de las resistencias antimicrobianas según el lugar de estancia hospitalaria, se observó que los pacientes con antecedente de internación en UCI presentaron una mayor resistencia a imipenem. Además, se constató mayor frecuencia de ITU-C en esta área, al compararlos con los pacientes internados en sala general. Estas diferencias en los perfiles de resistencia antimicrobianas entre ITU-C e ITU-noC, podrían atribuirse al antecedente de estancia en unidades críticas y no exclusivamente a la presencia de catéter urinario.

Colistín es considerado como tratamiento de última línea para microorganismos multi-resistentes responsables de infecciones asociadas a la atención de la salud. Debido a la emergencia de incremento de resistencia plasmídica en los últimos años, en Argentina se ha decidido incluir este antimicrobiano dentro de los utilizados para pacientes con ITU, tanto adquiridos en la comunidad como intrahospitalarios $^{31}$. En nuestro hospital, dicho antimicrobiano tuvo una actividad in vitro de $100 \%$ en la totalidad de los bacilos gramnegativos aislados, convirtiéndolo en una herramienta efectiva para el tratamiento de infecciones por microorganismos multi-resistentes.

Este estudio presenta las limitaciones de ser retros- pectivo, unicéntrico, y de haberse realizado a partir de urocultivos positivos y no a partir de la población total de pacientes internados. Por dichos motivos, no fue posible calcular las tasas de infección. Sin embargo, al disponer de la totalidad de los urocultivos positivos de nuestro centro, se pudo identificar todas las infecciones urinarias asociadas a atención de la salud y observar su comportamiento en situaciones clínicas diferentes a las descritas clásicamente, como internación en UCI y asociadas a catéteres urinario permanente.

Como conclusión, podemos afirmar que, en el presente estudio, las ITU-noC tuvieron una frecuencia no despreciable dentro de la totalidad de las ITU-AAS. Así mismo, los factores de riesgo de los pacientes con ITU-AAS, los agentes etiológicos responsables y sus correspondientes espectros de susceptibilidad, fueron similares en los grupos de ITU-C e ITU-noC. La vigilancia de las ITU-noC podría ayudar a identificar y controlar adecuadamente las ITU nosocomiales en distintas áreas del hospital. Además, su frecuencia debería hacer que los esfuerzos en materia de prevención, vigilancia y elaboración de guías de práctica clínica sobre ITU-AAS incluyan un análisis más allá de la cateterización urinaria y la internación en UCI.

\section{Resumen}

Introducción: Las infecciones urinarias asociadas a la atención de la salud (ITU-AAS) representan un importante problema sanitario, siendo poco conocidas sus características cuando no están asociadas a cateterización urinaria u ocurren fuera de unidades de cuidados intensivos (UCI). Objetivos: Determinar las características de los pacientes con ITU-AAS, etiología y susceptibilidad antimicrobiana de las mismas, tanto asociadas a catéter (ITU-C) como no asociadas a catéter (ITU-noC), en UCI y en sala general. Materiales y Métodos: Se realizó un estudio analítico retrospectivo de corte transversal entre 2009 y 2013 en un hospital universitario de tercer nivel. Se identificaron todos los episodios de ITU-AAS, diferenciándolas en ITU-C e ITU-noC. Resultados: Se incluyeron 253 episodios de ITU-AAS, siendo más frecuentes las ITU-C $(60,9 \%)$ respecto a ITU-noC. Un $37,4 \%$ de ITU-noC y $59,7 \%$ de ITU-C ocurrieron en UCI. Los microorganismos aislados más frecuentemente fueron Escherichia coli, Klebsiella pneumoniae y Enterococcus sp. El 19\% de los bacilos gramnegativos fueron productores de $\beta$-lactamasa de espectro extendido, siendo su frecuencia similar en ambos grupos. Conclusión: Las co-morbilidades de los pacientes con ITU-AAS, los agentes etiológicos responsables y sus correspondientes espectros de sensibilidad, fueron similares en los grupos de ITU-C e ITU-noC, tanto en sala general como en UCI. 


\section{Referencias bibliográficas}

1.- Chenowth C E, Saint S. Urinary tract infections. Infect Dis Clin North Am 2011; 25 (1): 103-15. doi: 10.1016/j.idc.2010.11.005. Epub 2010 Dec 18.

2.- Hooton T M, Bradley S F, Cardenas D D, Colgan R, Geerlings S E, Rice J C, et al. Infectious Diseases Society of America. Diagnosis, prevention, and treatment of catheter-associated urinary tract infection in adults: 2009 International Clinical Practice Guidelines from the Infectious Diseases Society of America. Clin Infect Dis 2010; 50 (5): 625-63.

3.- Iacovelli V, Gaziev G, Topazio L, Bove P, Vespasiani G, Finazzi Agrò E. Nosocomial urinary tract infections: A review. Urología 2014; 81 (4): 222-7. doi: 10.5301/uro.5000092. Epub 2014 Nov 12.

4.- Nicolle L E. Urinary catheter-associated infections. Infect Dis Clin North Am 2012; 26 (1): 13-27. doi: 10.1016/j.idc.2011.09.009.

5.- Pigrau C. Nocosomial urinary tract infections. Enferm Infecc Microbiol Clin 2013; 31 (9): 614-24. doi: 10.1016/j.eimc.2012.11.015. Epub 2013.

6.- Weber D J, Sickbert-Bennett E E, Gould C V, Brown V M, Huslage K, Rutala W A. Incidence of catheter-associated and noncatheter-associated urinary tract infections in a healthcare system. Infect Control Hosp Epidemiol 2011; 32 (8): 822-3. doi: 10.1086/661107.

7.- Nouetchognou J S, Ateudjieu J, Jemea B, Mesumbe E N, Mbanya D. Surveillance of nosocomial infections in the Yaounde University Teaching Hospital, Cameroon. BMC Res Notes [Internet]. BioMed Central 2016; 9 (1): 505. doi: 10.1186/s13104-016-2310-1.

8.- Trautner B W: Management of catheterassociated urinary tract infection. Curr Opin Infect Dis 2010; 23 (1): 76-82. doi: 10.1097/ QCO.0b013e328334dda8.

9.- Lewis S S, Knelson L P, Moehring R W, Chen L F, Sexton D J, Anderson D J. Comparison of non-intensive care unit (ICU) versus ICU rates of catheter-associated urinary tract infection in community hospitals. Infect Control Hosp Epidemiol 2013; 34 (7): 744-7. doi: 10.1086/671000. Epub 2013 May 22.

10.- Isikgoz Tasbakan M, Durusoy R, Pullukcu H, Sipahi O R, Ulusoy S, 2011. Turkish Nosocomial Urinary Tract Infection Study Group. Hospital-acquired urinary tract infection point prevalence in Turkey: differences in risk factors among patient groups. Ann Clin Microbiol Antimicrob 2013; 12: 31. doi: 10.1186/1476-0711-12-31.

11.- Bouza E, San Juan R, Muñoz P, Voss A, Kluytmans J. Co-operative Group of the European Study Group on Nosocomial Infections. A European perspective on nosocomial urinary tract infections II. Report on incidence, clinical characteristics and outcome (ESGINI-04 study). European Study Group on Nosocomial Infection. Clin Microbiol Infect 2001; 7 (10): 532-42.

12.- Chen L, Ou T, Teng S, Chen F, Hsieh T, Lee W. Hospital-acquired urinary tract infections in patients with diabetes and urinary catheterization. J Exp Clin Med 2014; 6 (3): 90-3.

13.- Lossa G R, Giordano Lerena R, Fernández L E, Vairetti J, Díaz C, Arcidiácono D, et al. Prevalence of hospital infections in adult intensive care units in Argentina. Rev Panam Salud Pública 2008; 24 (5): 324-30.

14.- Rosenthal V D, Guzmán S, Crnich C. Deviceassociated nosocomial infection rates in intensive care units of Argentina. Infect Control Hosp Epidemiol 2004; 25 (3): 251-5. DOI: $10.1086 / 502386$.

15.- Rossi A, Tokumoto M, Galas M, Soloaga R, Corso A. Monitoring antibiotic resistance in Argentina. The WHONET program, 19951996. Rev Panam Salud Pública 1999; 6 (4): 234-41.

16.- López M J, Cortés J A. Urinary tract colonization and infection in critically ill patients. Med Intensiva 2012; 36 (2): 14351. doi: 10.1016/j.medin.2011.06.007. Epub 2011 Aug 11.

17.- Horan T C, Andrus M, Dudeck M A. CDC/ NHSN surveillance definition of health careassociated infection and criteria for specific types of infections in the acute care setting. Am J Infect Control 2012; 36 (5): 309-32. doi: 10.1016/j.ajic.2008.03.002.

18.- Horan T C, Gaynes R P. Surveillance of nosocomial infections. In: Mayhall C G, editor. Hospital Epidemiology and Infection Control. 3rd ed. Philadelphia: Lippincott Williams \& Wilkins 2004; 1659-702.

19.- Hooton T M, Stamm W E. Diagnosis and treatment of uncomplicated urinary tract infection. Infect Dis Clin North Am 1997; 11 (3): 551-81.

20.- Grabe M, Bartoletti R, Bjerklund Johansen T E, Cai T, Çek M, Köves B, et al. Guidelines on urological Infections. 2015. Eur Assoc Urol 2015 [(accessed on 25 July 2017)]. Available online: http://uroweb.org/wpcontent/uploads/19-Urological-infections_LR2. pdf.

21.- Tenke P, Kovacs B, Bjerklund Johansen T E, Matsumoto T, Tambyah P A, Naber K G. European and Asian guidelines on management and prevention of catheter-associated urinary tract infections. Int J Antimicrob Agents 2008; 31 (Suppl 1): S68-78.

22.- Lu P L, Liu Y C, Toh H S, Lee Y L, Liu Y M, Ho C M, et al. Epidemiology and antimicrobial susceptibility profiles of Gram-negative bacteria causing urinary tract infections in the Asia-Pacific region: 2009-2010 results from the Study for Monitoring Antimicrobial
Resistance Trends (SMART). Int J Antimicrob Agents 2012; 40: 37-43. doi: 10.1016/S09248579(12)70008-0.

23.- Redder J D, Leth R A, Møller J K. Analysing risk factors for urinary tract infection based on automated monitoring of hospital-acquired infection. J Hosp Infect 2016; 92 (4): 397-400. doi: 10.1016/j.jhin.2015.12.009. Epub 2015 Dec 30.

24.- Cairns S, Reilly J, Booth M. Prevalence of healthcare-associated infection in Scottish intensive care units. J Hosp Infect 2010; 76 : 308-10. doi: 10.1016/j.jhin.2010.05.010. Epub 2010 Oct 23.

25.- Córdova E, Lespada M I, Cecchini D, Jacob N, Gómez N, Gutfraind G. Prevalencia de gérmenes multirresistentes en infecciones del tracto urinario de la comunidad y asociadas a los cuidados de la salud. Actualizaciones en SIDA e Infectología 2014; 22: 33-8.

26.- Hennequin C, Robin F. Correlation between antimicrobial resistance and virulence in Klebsiella pneumoniae. Eur J Clin Microbiol Infect Dis. 2016; 35 (3): 333-41. doi: 10.1007/ s10096-015-2559-7. Epub 2015 Dec 30.

27.- Bouchillon S K, Badal R E, Hoban D J, Hawser S P. Antimicrobial susceptibility of inpatient urinary tract isolates of gram-negative bacilli in the United States: Results from the Study for Monitoring Antimicrobial Resistance Trends (SMART) Program: 2009-2011. Clin Ther 2013; 35(6): 872-7. doi: 10.1016/j. clinthera.2013.03.022. Epub 2013 Apr 25.

28.- Fasugba O, Gardner A, Mitchell B G, Mnatzaganian G. Ciprofloxacin resistance in community-and hospital-acquired Escherichia coli urinary tract infections: a systematic review and meta-analysis of observational studies. BMC Infect Dis 2015; 15 (1): 545. doi: 10.1186/s12879-015-1282-4.

29.- Ma K L, Wang C X. Analysis of the spectrum and antibiotic resistance of uropathogens in vitro: Results based on a retrospective study from a tertiary hospital. Am J Infect Control 2013; 41 (7): 601-6. doi: 10.1016/j. ajic.2012.09.015. Epub 2013 Jan 23.

30.- Hoban D J, Lascols C, Nicolle L E, Badal R, Bouchillon S, Hackel M, et al. Antimicrobial susceptibility of Enterobacteriaceae, including molecular characterization of extendedspectrum beta-lactamase-producing species, in urinary tract isolates from hospitalized patients in North America and Europe: results from the SMART study 2009-2010. Diagn Microbiol Infect Dis 2012; 74 (1): 62-7. doi: 10.1016/j. diagmicrobio.2012.05.024. Epub 2012 Jul 2.

31.- Rapoport M, Faccone D, Pasteran F, Ceriana P, Albornoz E, Petroni A, et al. First description of mcr-1-mediated colistin resistance in human infections caused by Escherichia coli in Latin America. Antimicrob Agents Chemother 2016; 60 (7): 4412-3. doi: 10.1128/AAC.00573-16. Print 2016 Jul. 